\title{
Orientation Gradients in Relation to Grain Boundaries at Varying Strain Level and Spatial Resolution
}

\author{
Samikshya Subedi ${ }^{\mathrm{a}, *}$, Reeju Pokharel ${ }^{\mathrm{a}, \mathrm{b}}$, Anthony D. Rollett ${ }^{\mathrm{a}}$ \\ ${ }^{a}$ Department of Materials Science and Engineering, Carnegie Mellon University, 5000 Forbes Avenue Pittsburgh PA 15213, USA \\ ${ }^{b}$ Materials Science and Technology Division, Los Alamos National Laboratory, Los Alamos, NM 87544, USA
}

\begin{abstract}
The resolution dependence of orientation gradients was studied in a well-annealed $99.9995 \%$ pure polycrystalline copper pulled to failure in tension. Owing to the well developed neck, different regions in the sample correspond to different tensile strains. Post-mortem characterization was performed using EBSD on cross-sections containing the tensile axis. Kernel average misorientation (KAM) was calculated as a metric to establish correlation between defect accumulation and microstructural features, with a threshold of $5^{\circ}$ to focus on intra-granular gradients. The region with the lowest strain $(2 \%)$ showed high KAM values adjacent to grain boundaries compared to the grain interior, regardless of the point spacing, i.e. the spatial resolution. However, in the region with the highest strain $(13 \%)$ a strong dependence on resolution was found. For point spacings of $0.5 \mu \mathrm{m}$ or smaller, the same correlation of high KAM with locations near boundaries was found. At coarse spacings i.e. low spatial resolution, by contrast, the reverse was found in that the highest KAM values appear in the grain interiors, as previously observed in x-ray microscopy on the same sample which had a similar coarse resolution. An analysis of orientation gradients parallel to, and perpendicular to boundaries suggested that the latter tend to be the larger of the two. This helps to explain why boundary-adjacent points have low KAM values. The conclusion is that measurement of local orientation gradient requires a resolution that is comparable to the dislocation substructure.
\end{abstract}

Keywords: KAM; EBSD; Grain Boundaries; Step size; Strain

\section{Introduction}

\subsection{Brief overview of orientation gradients}

Polycrystalline metals subjected to external load (plastic deformation) respond by straining in ways that are heterogeneous at several length scales. At the grain scale one such response is the development of "in-grain" orientation gradients or dispersion in the change in orientation (i.e. texture development) of the grain at a local level. The theory behind this change is that spatial gradients in stress, e.g. between grains, during deformation leads to variations in which (multiple) slip systems are active at any given point within a grain,

\footnotetext{
${ }^{*}$ Corresponding author.

Email address: ssubedi@andrew.cmu.edu (Samikshya Subedi)
} 
the accumulation of which results in orientation gradients [1, 2, 3, 4, 5. This behavior is highly dependent on the initial orientation of a grain with respect to the tensile axis as well as its interaction with neighboring grains [6, 7, 8]. One of the reasons to investigate orientation gradients is that they can be directly associated with the amount of applied plastic strain [9, 10, enabling quantification of plastic deformation in materials. For instance, knowing how the gradients are locally spread in a grain will help the analysis of crack initiation and propagation in metals [11].

Orientation gradients may be correlated to geometrically necessary dislocations (GNDs), which are generated to accommodate local incompatibilities in crystallographic parameters. It has been shown that higher misorientation corresponds to higher defect accumulation in polycrystals and thus larger GNDs density [2, 10.

Thanks to the steady accumulation of orientation gradients, grains reorient and subdivide at high strains into smaller grains or crystallites [12], in order to accommodate macroscopically imposed strain. Local variation in orientations and grain fragmentation also influence the overall texture and finally mechanical properties in metals [8]. Orientation also plays a significant role in the generation of new grains during recrystallization; generally recrystallization nucleates in regions of large orientation gradients [10, 13].

\subsection{Characterizing orientation gradients}

Plastic deformation in polycrystals at mesoscale is known to be heterogeneous, which can be attributed in part to crystallographic anisotropy exhibited by individual grains and in part to the tendency of dislocations to be stored in a non-uniform manner. Diffraction microscopy methods can provide spatially resolved discrete orientation maps either from surface (Electron Back-Scatter Diffraction, EBSD) or bulk (near-field HighEnergy Diffraction Microscopy, nf-HEDM) of the sample. This discrete data provides an opportunity to investigate orientation gradient development and orientation change during plastic deformation at a local level. Orientation gradient calculation is intimately related to the spatial resolution of the measured orientation maps. In order to quantify the relationship between mechanical properties and plastic deformation, it is important to perform a systematic study of the spatial resolution dependence of orientation gradients. A previous study utilized the nf-HEDM method to study spatially-resolved microstructure evolution in $\mathrm{Cu}$, where lattice rotation, orientation change, and orientation gradient development were reported up to $14 \%$ tensile strain [1, 14]. Experimental observations were compared with a crystal plasticity model. Experimental results illustrated high KAM values near boundary at lower strain levels, while at larger strains, a broader distribution in KAM values was observed away from the grain boundaries. However, computer simulations (using FFT based visco-plastic simulation (VPFFT)), high KAM values were predicted near grain boundaries [1, 14. The latter is an expected phenomenon since dislocations generally accumulate at the grain boundary (GB) 3, 15, 16. This significant discrepancy between experiment and simulation highlights the importance of conducting a thorough experiment, to test the dependence of KAM on step size.

One possible explanation of this discrepancy is an inadequate spatial resolution in nf-HEDM. In this particular study, the spatial resolution of the orientation maps obtained from nf-HEDM scans was $2.8 \mu \mathrm{m}$. 
However, the size of the subgrain structures for copper is known to be at a submicron length scale 11, 14, 16, 17. It is hypothesized that the step size used in HEDM was too coarse to capture sub-grain features in copper. Kamaya et al. 9] stated that the orientation spread depends on "the degree of plastic strain but not on the data density of the crystal orientation map," while Takamaya et al. [10, 18, reports that "the magnitude of KAM is affected by step size." Similarly, Wright et al. [19] also clearly mentions that KAM is highly sensitive to the step size. Furthermore, Li observed "sharpening of boundaries" and "convergence" of KAM features with increasing spatial resolution [20].

In this work, we have used KAM as a metric for quantifying local orientation gradient and investigated the resolution dependance on KAM calculation. EBSD allows for finer resolution for mapping crystal orientation at approximately $50 \mathrm{~nm}$ spatial resolution [21. This work was performed on the same sample of copper on which HEDM was performed. A resolution study at different strain levels was carried out to see how the experimental KAM behavior changes in relation to grain boundaries when step sizes are changed and a direct comparison between the two methods is presented in this study.

\section{Materials and Experimental Method}

For this experiment, a highly annealed $99.9995 \%$ pure copper specimen, having a $1 \mathrm{~mm}$ diameter gauge section (shown in Figure 1a), was pulled to failure in tension and cross-sectioned parallel to the tensile axis. The sample was polished on a mechanical polisher using 600, 800, and 1200 grit $\mathrm{SiC}$ paper for about 3 minutes each in order to remove the surface roughness of the sample. Next, 9,3 and $1 \mu \mathrm{m}$ diamond polishing was performed using an oil-based suspension for 5 minutes. Final polishing was done using colloidal silica slurry for a high quality surface finish for EBSD. A fiducial mark was made in order to have an estimate of where the scans were being taken.

Once the sample was prepared, EBSD scans were taken using EDAX/TSL acquisition software with a Hikari EBSD detector on a Quanta 200 FEG SEM. The microscope settings used were an accelerating voltage of $20 \mathrm{KV}$, a spot size of 5 and an aperture size $5.150 \times 150 \mu \mathrm{m}$ square grid scans were taken at regions of different strain level. Step sizes were varied from $2.5,1,0.5$ and $0.2 \mu \mathrm{m}$ at both high strain and low strain scans. Each of these maps was cleaned using the single dilation cleanup with the single iteration option. Further analysis on KAM spread was done using built-in KAM functions in TSL. 


\section{Results and Discussion}

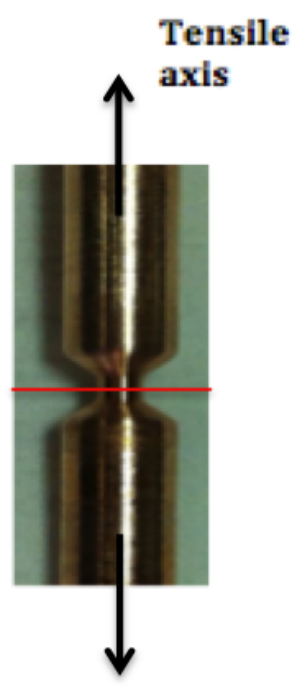

(a)

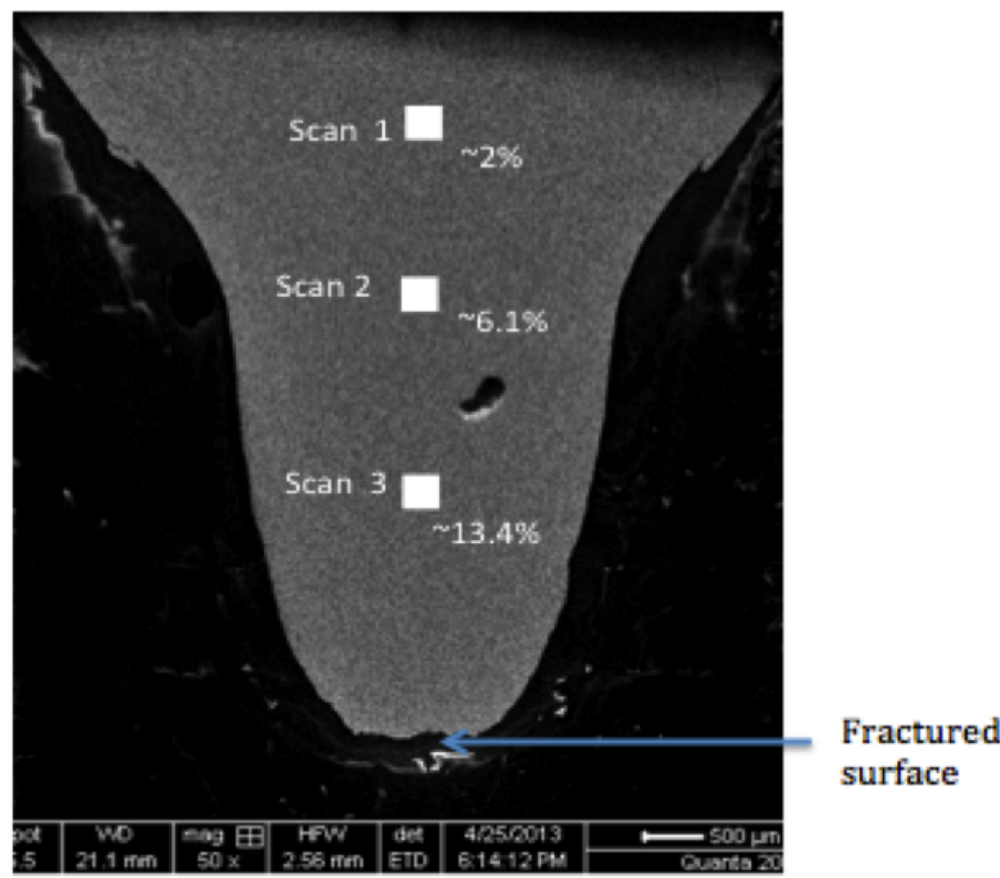

(b)

Figure 1: a) Copper sample with a cylindrical gauge section of $1 \mathrm{~mm}$ diameter and $1 \mathrm{~mm}$ height. b) Cross-sectioned gauge section showing three different scan regions with their respective estimated strain. A fiducial mark (between 2nd and 3rd mark) was made to aid in locating and orienting the EBSD scans relative to the low magnitude images of the cross-section.

As shown in Figure 10), EBSD maps were taken at three scan regions, with each having a different level of plastic strain. Scan 3 (closest to the fractured surface) clearly has high strain while Scan 1 (farthest from the fractured surface) has comparatively low strain. Change in diameter at each scan region were recorded in order to estimate the amount of strain. It should be noted that while grinding the gauge section care was taken to ensure that the section was in plane relative to the centerline. The initial diameter of the gage section was $1000 \mu \mathrm{m}$. The final diameters at region 1-3 were measured to be 990, 970, and $935.1 \mu \mathrm{m}$ respectively. The strain at each point was thus estimated to be $2 \%, 6.1 \%$ and $13.4 \%$ by using the strain formula for diameter:

$$
\text { True } \operatorname{strain}=\ln \left(\frac{A_{o}}{A}\right)=\ln \left(\frac{d_{o}^{2}}{d^{2}}\right)
$$

Here $A_{\circ}$ is the initial and $A$ is the current cross-sectional area. Similarly, $d_{\circ}$ is the initial and $d$ is the local diameter.

After the approximate strain was calculated at each scan point, misorientation distributions were examined. 


\subsection{KAM behavior dependent on strain}

Orientation maps were generated for the three selected regions shown in Figure 1 1 ). The initial EBSD scan was performed using a $2.5 \mu \mathrm{m}$ step size, which is similar to the spatial resolution used in the nf-HEDM study. Inverse pole figure (IPF) maps are plotted for scans 1-3, which are shown in Figure 2a. From the IPF maps, it is obvious that the grains are elongated parallel to the tensile axis with increasing strain. Misorientation development within the grain can be attributed to the increase in dislocation density [9, 17, 22. This increase is evident through a decrease in average confidence index (CI) and image quality (IQ) values of the measured Kikuchi pattern. CI value is an indication of how accurate the indexing is and ranges from $0-1,0$ being the lowest and 1 being the highest. IQ is a measure of the sharpness of the Kikuchi bands where higher IQ values imply a better pattern quality [22].

The KAM of each point is calculated as the average misorientation angle between that point and its specified number of nearest neighbor or pixels (in 2D) and voxels (in 3D) 1, 2]. KAM was calculated from 2D orientation maps up to the second nearest neighbors. Any contributions to the KAM that exceeded a chosen threshold value, in this case $5^{\circ}$, were excluded assuming they correspond to a grain boundary. KAM maps as shown in Figure 2b) (given by orientation imaging microscopy (OIM) analysis software) illustrate that the average KAM value increases with increasing strain. In general, as the plastic strain increases, dislocation density and KAM accumulation in the microstructure increases, with corresponding decreases of average CI and IQ.

At lower strains, higher KAM values seem to be localized near some grain boundaries, but no obvious trend can be seen from the KAM maps in Figure $2 \mathrm{~b}$ ). In order to quantify the relationship between orientation gradient and microstructural features, a more statistical approach was taken by correlating KAM and distance from grain boundary (GB). For this, a Euclidean distance map [5, 16] was computed based on the distance from the nearest grain boundary. For the pair of variables, one variable was chosen as the independent variable (distance from GB) and the values of the other variable (KAM) were binned against it [5]. Thus, the average KAM against distance from GB was plotted. The binning process could be repeated for the complementary choice of independent variable.

From Figure 22) it is clear that, at low strain, the largest KAM values occur close to grain boundaries. On average, KAM decreases with increasing distance from a boundary towards the interior of a grain. Counterexamples of this overall trend, however, were found in the high strain region. Here large KAM values were observed inside the grain, which is, in fact, consistent with what was observed with the HEDM study. With increasing distance from GB, KAM first increases and then falls off. This behavior is not as expected but could be explained using grain fragmentation phenomenon which explains that there is a development of sub-grains structures at high plastic strains [12]. Thus step size becomes crucial since it needs to be fine enough to capture those sub-grain features inside the grain. 
a)

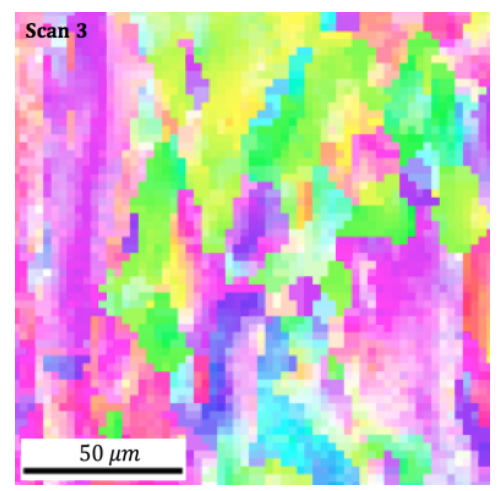

Average CI: 0.55

Average IQ: 1011.50

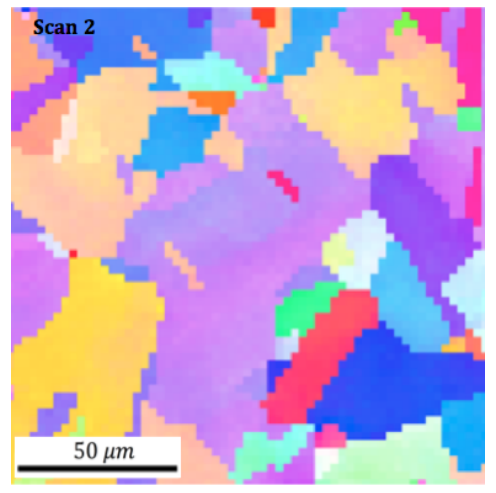

Average CI: 0.64

Average IQ: 1667.96
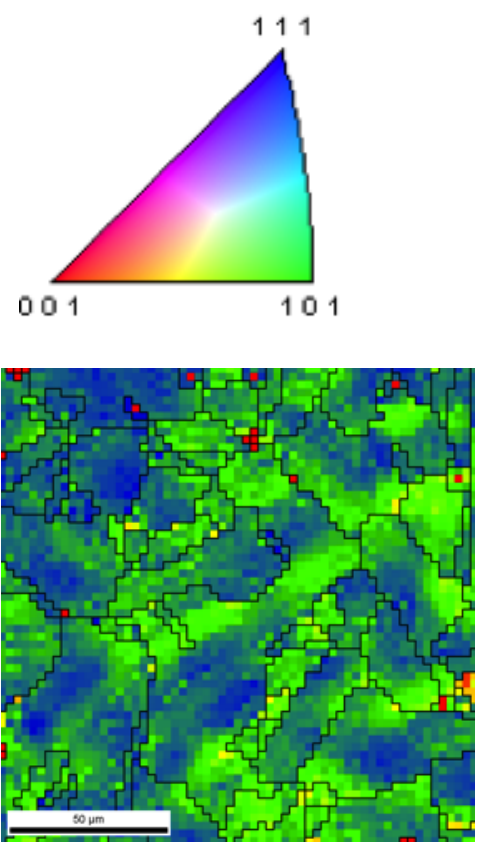

Average KAM: 0.86

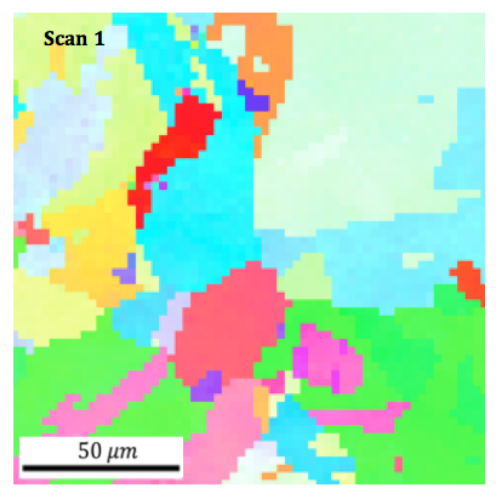

Average CI: 0.62

Average IQ: 1467.83

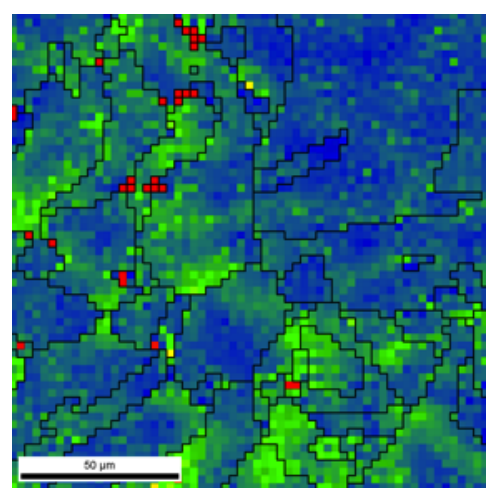

Average KAM: 0.64
Average KAM: 3.20

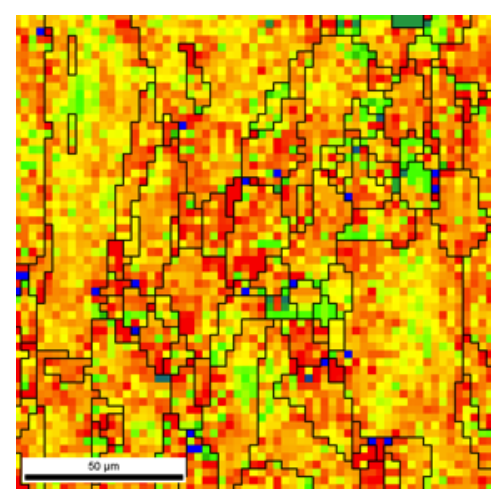

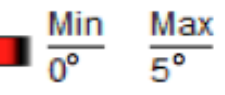

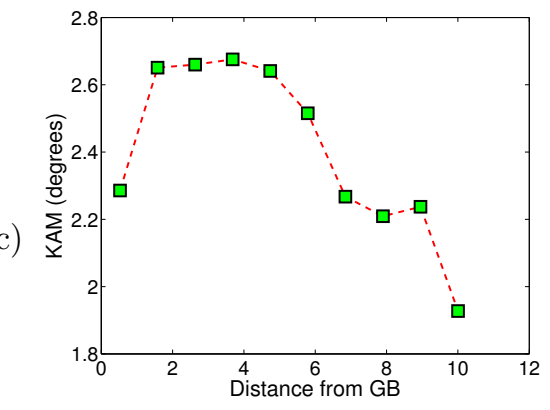

(i) $13.4 \%$

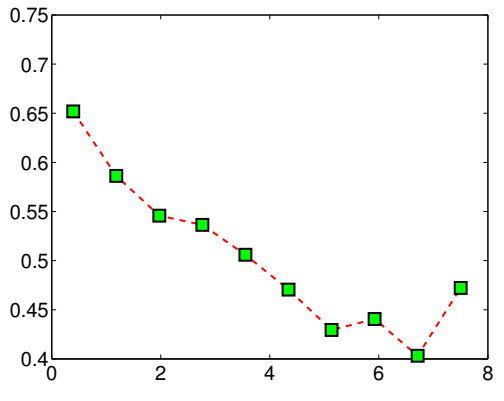

(ii) $6.1 \%$

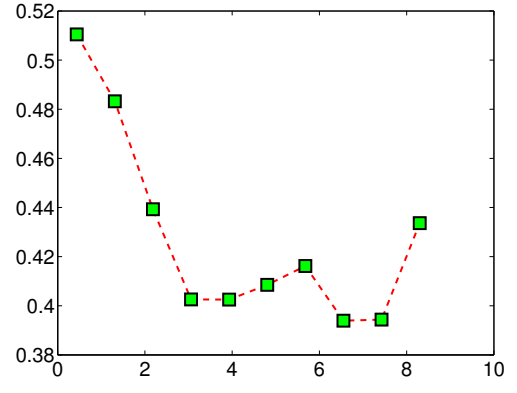

(iii) $2 \%$

Figure 2: a) IPF maps showing orientation mapping, average CI and IQ b) KAM map c) KAM vs. Distance from GB binned by distance. Each box represents a pixel. Thus the unit of distance is $2.5 \mu \mathrm{m}$ for a maximum of $25 \mu \mathrm{m}$ in the case of the righthand column. 


\subsection{KAM behavior dependent on step size/resolution}

A resolution study was performed at all three regions ('Scan 1', 'Scan 2' and 'Scan 3' respectively). Correlation plots were made to quantify the relationship between KAM and boundary distance as a function of spatial resolution/step size at varying strain levels. Scans 1 and 2 showed a similar lack of significant difference between $2.5 \mu \mathrm{m}$ and $0.5 \mu \mathrm{m}$ step size scans, as the strain was evidently not high enough to create very small sub-grain structures. We, therefore, report the details of only the 1st and 3rd scans.

\subsubsection{Low strain region ('Scan 1')}

For $2 \%$ strain, as shown in Figure 3i], the spatial resolution of the microstructure was refined by changing the step size from 2.5 to $0.5 \mu \mathrm{m}$. KAM maps were obtained at both resolutions in order to see if high KAM is localized in a particular region, specifically near grain boundaries. As shown in Figure 3ii), although some high KAM values (indicated by the traces of red and yellow colors) are seen at some grain boundaries, it is not clear that the KAM is localized at grain boundaries. Thus correlation plots were made for these corresponding microstructures. As shown in Figure 3iii for both resolutions, KAM is high at the boundary and gradually decreases towards the interior of the grains. This confirms that due to the coarse dislocation substructure at low strain the calculation of KAM is not sensitive to the step size used.

$2.5 \mu \mathbf{m}$

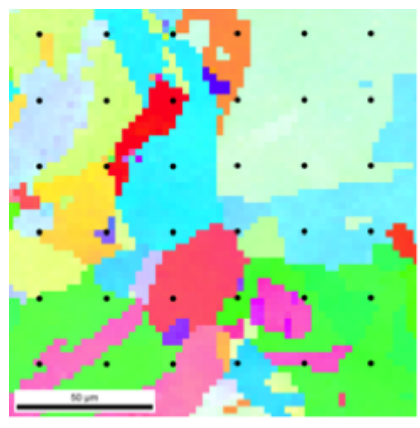

Average CI: 0.62

Average IQ: 1467.83

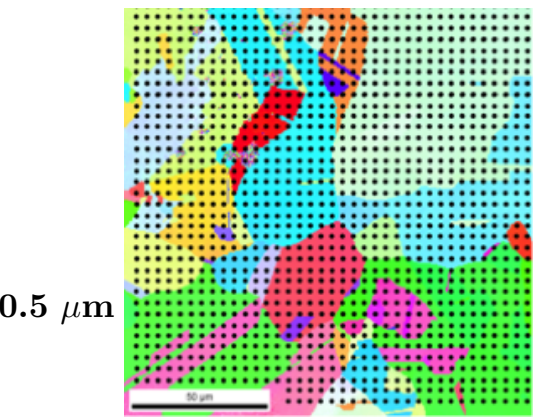

Average CI: 0.65

Average IQ: 1618.74

(i)
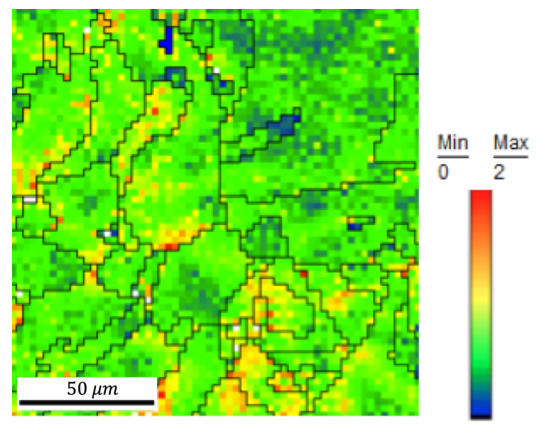

Average KAM: 0.65

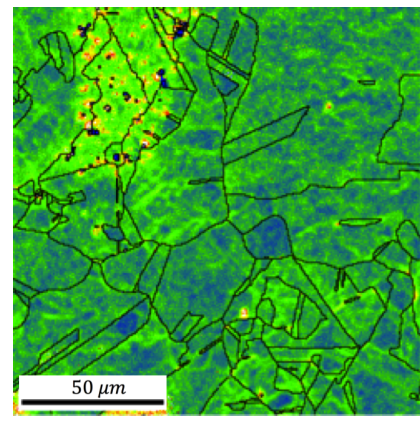

Average KAM: 0.44
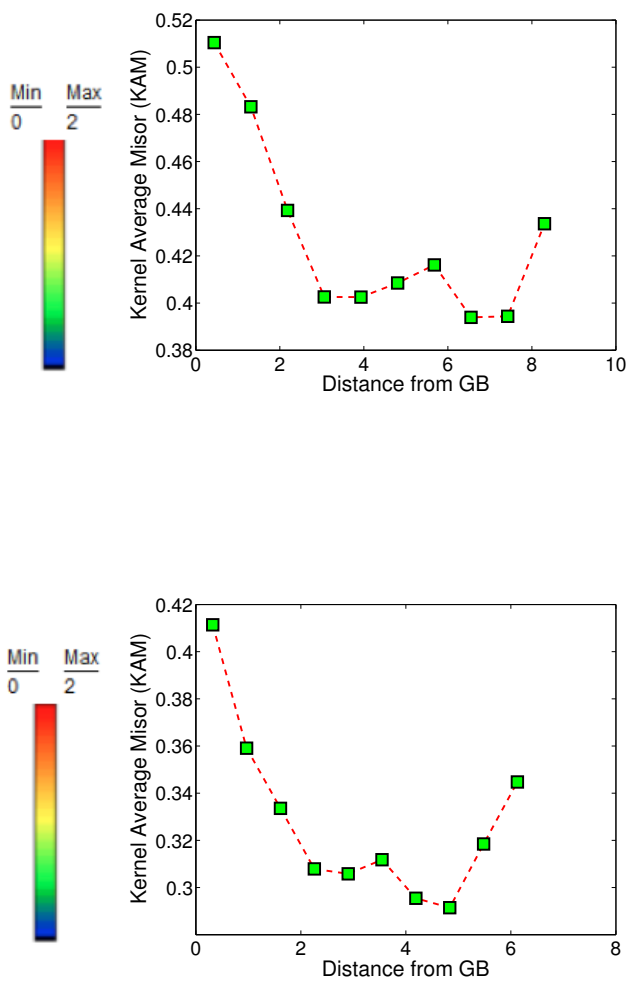

(iii)

Figure 3: i) IPF maps showing orientation mapping, black dotted points/grids are to show schematic of step size, average CI and IQ ii) KAM map iii) KAM vs. Distance from GB binned by distance 


\subsubsection{High strain region ('Scan 3')}

The resolution study at the high strain region was of the most interest as this is the region where the HEDM study was conducted and the inconsistency with the computational approach was observed. The highly strained region developed large misorientations and subgrain structures so it is essential to choose the correct step to do the KAM calculation lest incorrect conclusions be drawn. The results from the resolution study are reported in Figure 4,5 and 6 .

Scans with different resolution were taken in the $13.4 \%$ strain region by changing the step size from 2.5 to 1 to 0.5 to $0.2 \mu \mathrm{m}$. In this study, $2.5 \mu \mathrm{m}$ is considered to be a coarse step size, while a submicron step size is considered to be a fine step size. As a result, the nature of the graph changed as the step sizes were changed and the spatial resolution of the microstructure was refined with each consecutive scan as shown in Figure 4 a) and 4 b) below. The finer the resolution the more closely the graph approaches what was expected from previously published research; higher KAM at the GB and lower inside the grain (shown in Figure 4p) and d) [5, 9. Box plots (shown in Figure 5) were also made for these microstructures to show in more detail the distribution of orientation data at each bin, which very closely follows the same trend as earlier.

We note in passing the interesting feature, circled, of a grain with low KAM values that is divided by a twin-related grain with high KAM values. There is a weak dependence on orientation gradient [1] but the sharp difference evident in this location is more likely a consequence of the large local difference in orientation across the pair of twin boundaries. 
a)

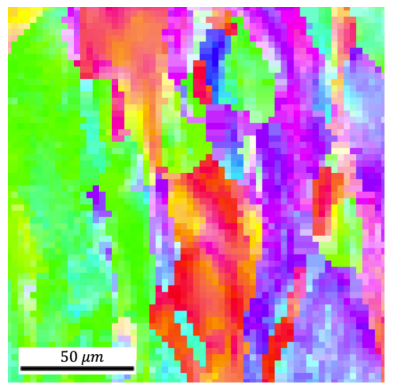

Average CI: 0.39

Average IQ: 920.95

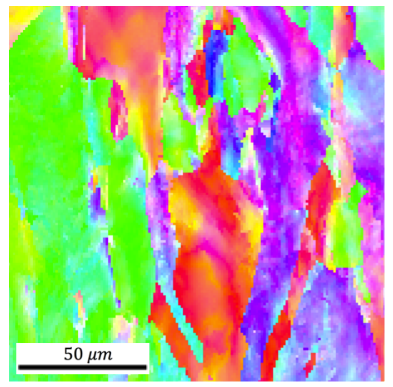

Average CI: 0.41

Average IQ: 981.7

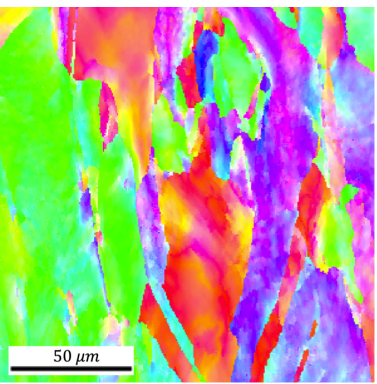

Average CI: 0.43

Average IQ: 1030.1

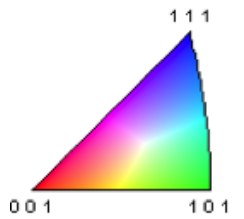

b)

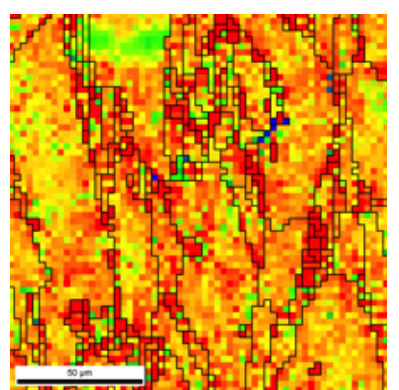

Average KAM: 3.39

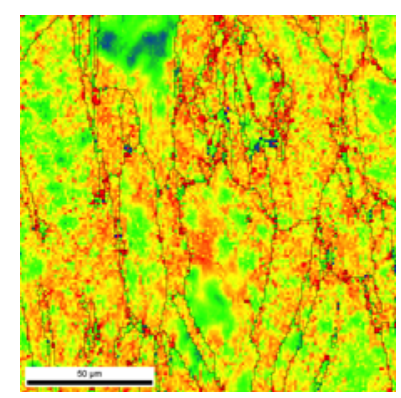

Average KAM: 2.66

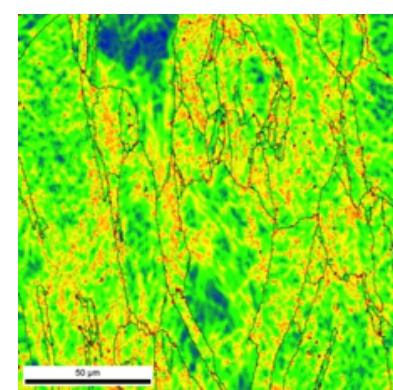

Average KAM: 1.93

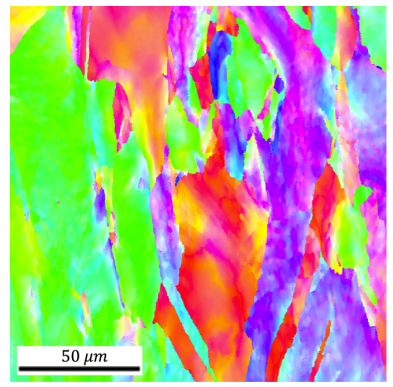

Average CI: 0.44

Average IQ: 1030.91

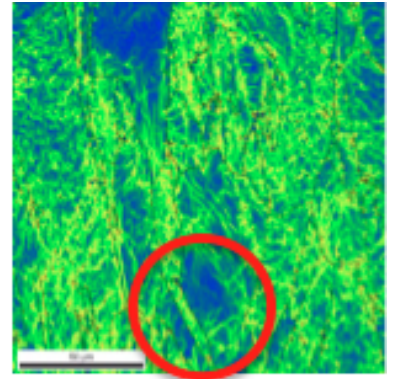

Average KAM: 1.16

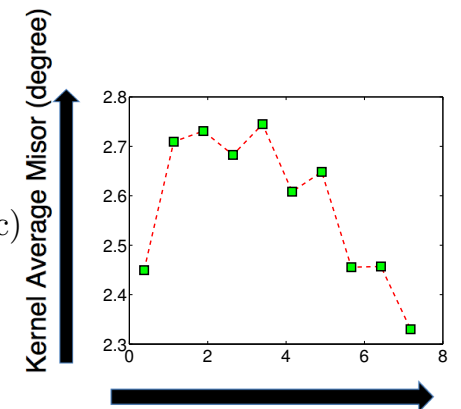

$\frac{\operatorname{Min}}{0^{\circ}} \frac{\operatorname{Max}}{5^{\circ}}$
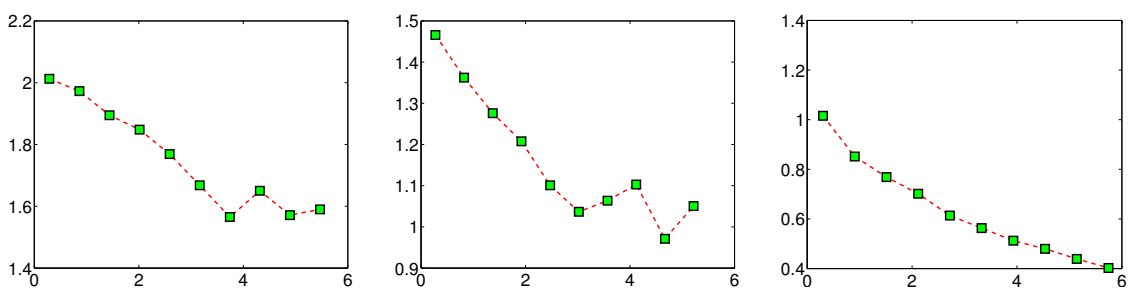

Distance from GB
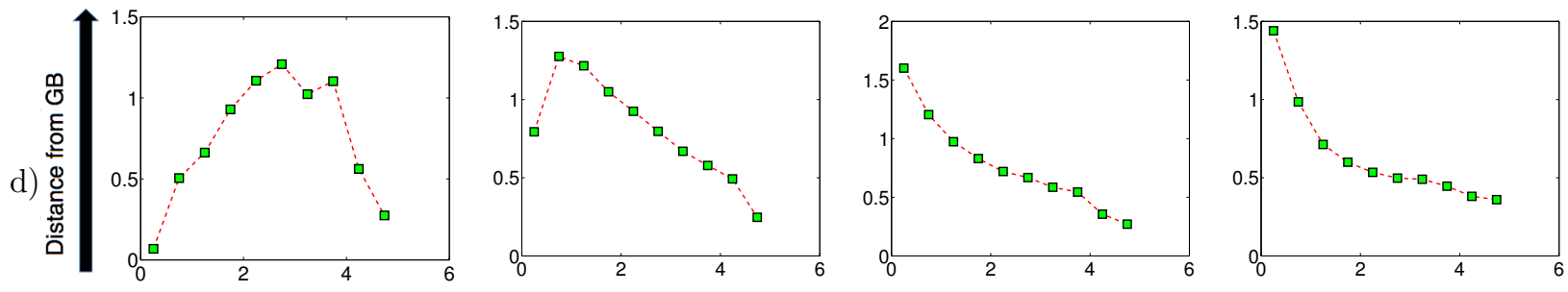

Kernel Average Misor (degree)

(i) $2.5 \mu \mathrm{m}$

(ii) $1 \mu \mathrm{m}$

(iii) $0.5 \mu \mathrm{m}$

(iv) $0.2 \mu \mathrm{m}$

Figure 4: a) IPF maps showing orientation mapping, average CI and IQ b) KAM maps c) KAM vs. Distance from GB binned by distance d) Distance vs. KAM binned by KAM for (i) $2.5 \mu \mathrm{m}$ ii $1 \mu \mathrm{m}$ (iii) $0.5 \mu \mathrm{m}$ (iv) $0.2 \mu \mathrm{m}$ scan respectively. 


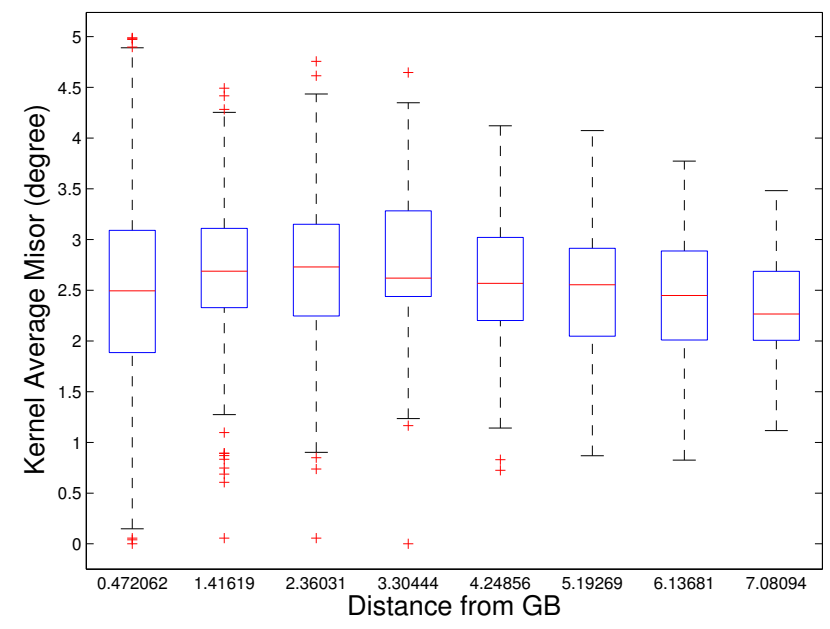

(a)

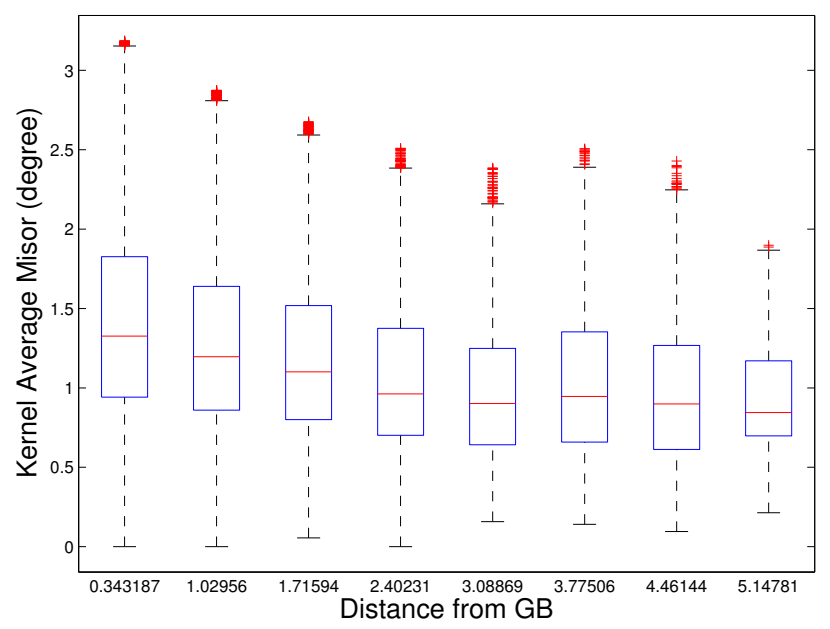

(c)

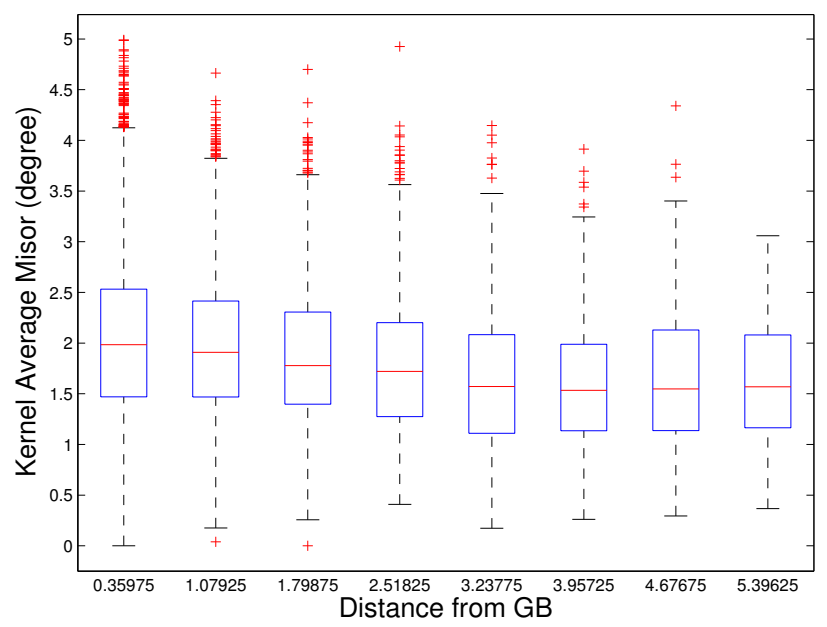

(b)

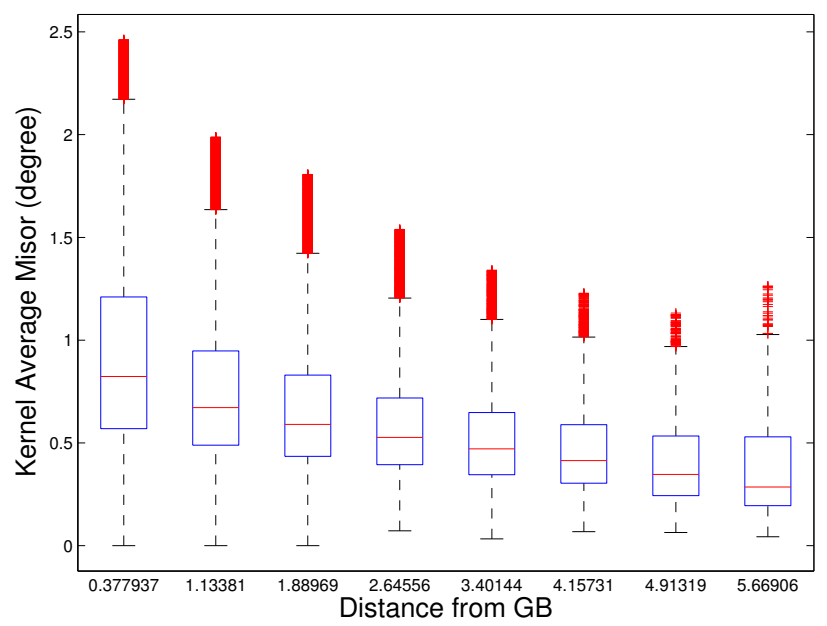

(d)

Figure 5: Box plots for different resolution microstructures i.e. a) $2.5 \mu \mathrm{m} \mathrm{b}) 1 \mu \mathrm{m} \mathrm{c)} 0.5 \mu \mathrm{m} \mathrm{d}) 0.2 \mu \mathrm{m}$ for $13.4 \%$ strain. Here, the red line is the median of the data, the upper blue line of the rectangular box is the 3rd quartile and the lower blue line is the 1st quartile. The differences between two is the interquartile range (IQR). Any data point more than 1.5 IQR above the third quartile and below the first quartile is an outlier, here denoted by a red cross.

As shown in the box plot for $2.5 \mu \mathrm{m}$ resolution, the trend closely follows what was observed in Figure 4 . A large number of outliers can be observed in the $0.2 \mu \mathrm{m}$ resolution box plot, but note that as the resolution is refined the number of points evaluated increases as the square of the linear point desnity. The $0.2 \mu \mathrm{m}$ dataset has 62.5 times as many data points as $2.5 \mu \mathrm{m}$ and 25 times as many as $1 \mu \mathrm{m}$. So the outliers that are shown in the plot are not significant compared to the number of data points being analyzed. The percentage of data that are outliers is $0.08 \%$ for $2.5 \mu \mathrm{m}, 0.81 \%$ for $1 \mu \mathrm{m}, 2.6 \%$ for $0.5 \mu \mathrm{m}$ and approximately $7 \%$ for $0.2 \mu \mathrm{m}$. 


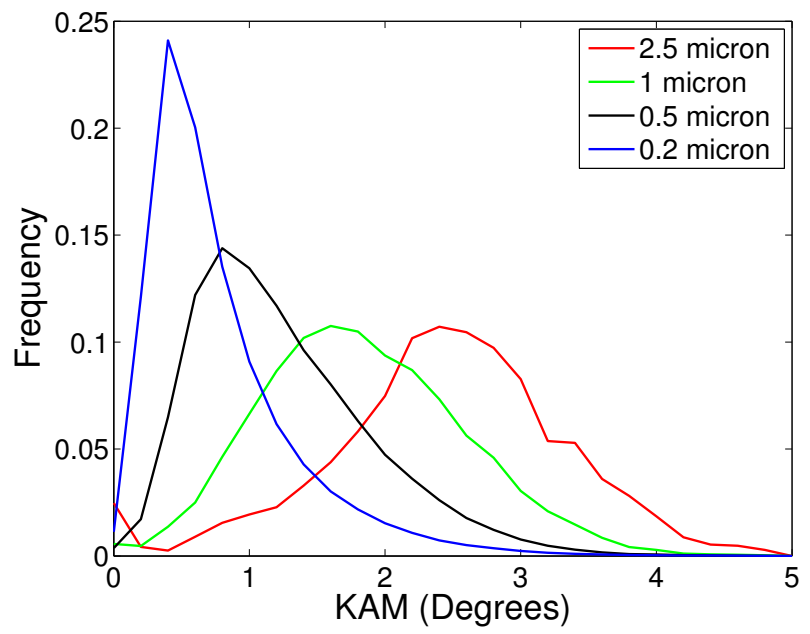

Figure 6: KAM distribution shown for different resolution scans, with step sizes given in the legend.

Similarly, frequency plots for different spatial resolution scans were also drawn in order to visualize the misorientation distribution for each resolution. The mdeian misorientation value is $\approx 2.5^{\circ}$ at a $2.5 \mu \mathrm{m}$ step size whereas it is $\approx 0.3^{\circ}$ at $0.2 \mu \mathrm{m}$. This drastic change in misorientation distribution with step size again emphasizes the strong influence of step size on KAM values.

\subsubsection{Sub-sampling}

In the $13.4 \%$ strain region, subsampling was also done on the $0.2 \mu \mathrm{m}$ step size scan to see whether the same result would be obtained as for varying the physical step size, i.e. the results shown in Figure 4. For this study the finest scan i.e $0.2 \mu \mathrm{m}$ scan was taken and alternate rows and columns were removed to coarsen the scan; in other words, to artificially increase the step size from $0.2 \mu \mathrm{m}$ to step sizes of $0.4,0.8,1.6,2.4 \mu \mathrm{m}$. Then the corresponding KAM values as a function of distance from GB and distance from GB as a function of KAM were plotted, as shown in Figure 7. The result demonstrated that the same variation in KAM distribution is found from sub-sampling as was obtained with physical variations in step size. 


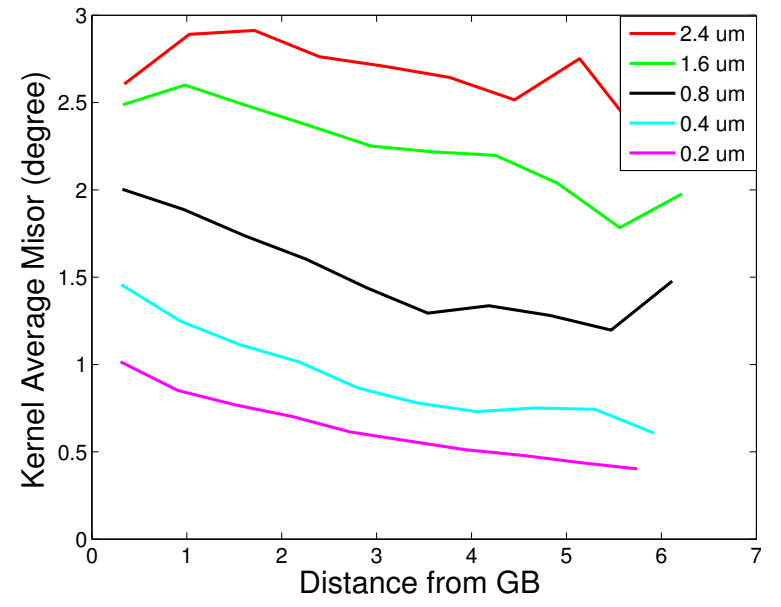

(a)

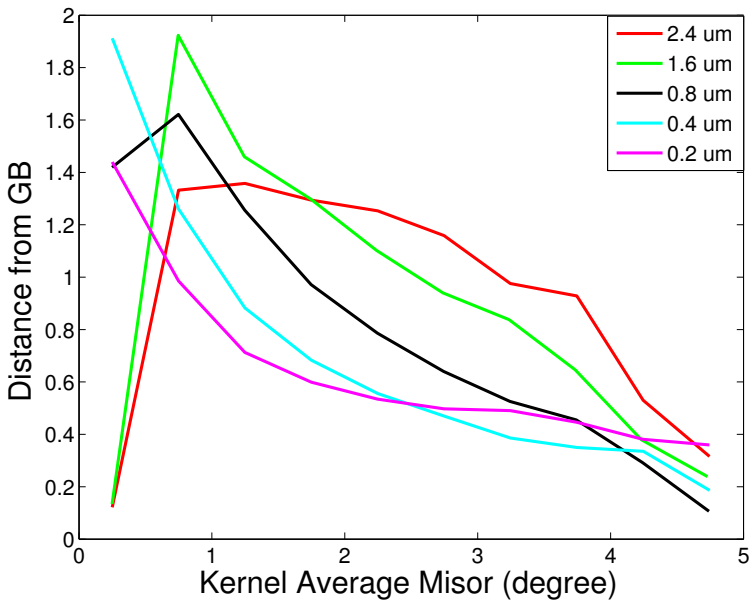

(b)

Figure 7: Sub-sampling results for a) KAM vs. distance b) Distance vs. KAM. The effective spatial resolution is shown in the legend.

\section{A model to rationalize the variation in the KAM-distance relationship with resolution}

In this section, we propose a simple model to rationalize the observation that lower KAM values are obtained near grain boundaries at coarse step sizes (above about a micron in the investigated copper). It is hypothesized that the orientation gradients perpendicular to the grain boundaries are larger than those parallel to GB. For a point adjacent to a grain boundary, such as the one shown in Figure 8, some of the points are naturally excluded because they lie outside the grain and have misorientations greater than the typical threshold of $5^{\circ}$. Out of the remaining points, if the orientation gradients parallel to the boundary are not as strong then, overall, the average KAM value decreases. 


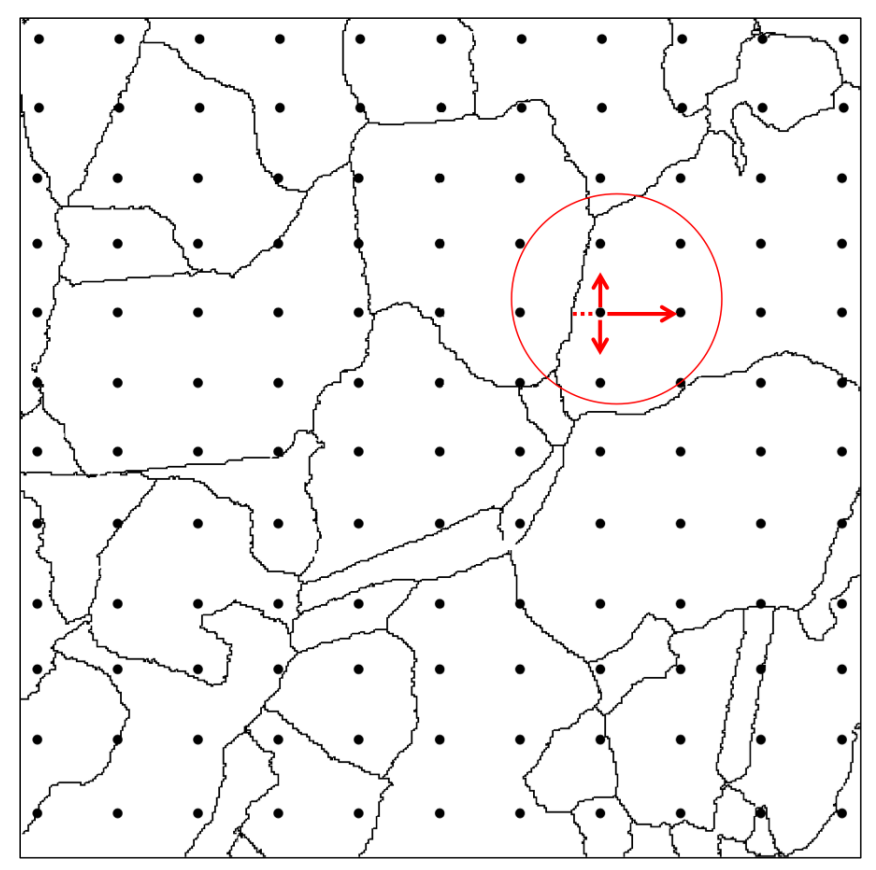

Figure 8: Schematics of KAM calculation at GB is shown, where dotted line represents the direction in which a neighboring point is ignored. A solid long arrow indicates a stronger gradient whereas a shorter arrow indicates a weaker gradient.

In order to assess the hypothesis quantitatively, multiple parallel and perpendicular traces of misorientation values relative to the initial point were extracted from the orientation maps. Average values of both these types of misorientation trace are plotted in Fig. 9 a. It is clear that the average misorientation value perpendicular to grain boundaries is larger than the average misorientation value parallel to grain boundaries, which supports the hypothesis. A typical example of what a perpendicular and parallel line plot for misorientation versus distance from GB is shown in Fig. 9p and c. We note that gradients in orientation near grain boundaries have been remarked on in the literature several times. Mishra et al., for example, measured enough gradients perpendicular to boundaries to make the case that larger gradients are found near boundaries than in the interior of grains [3]. The analysis of perpendicular versus parallel orientation gradients is, however, new to this work. 


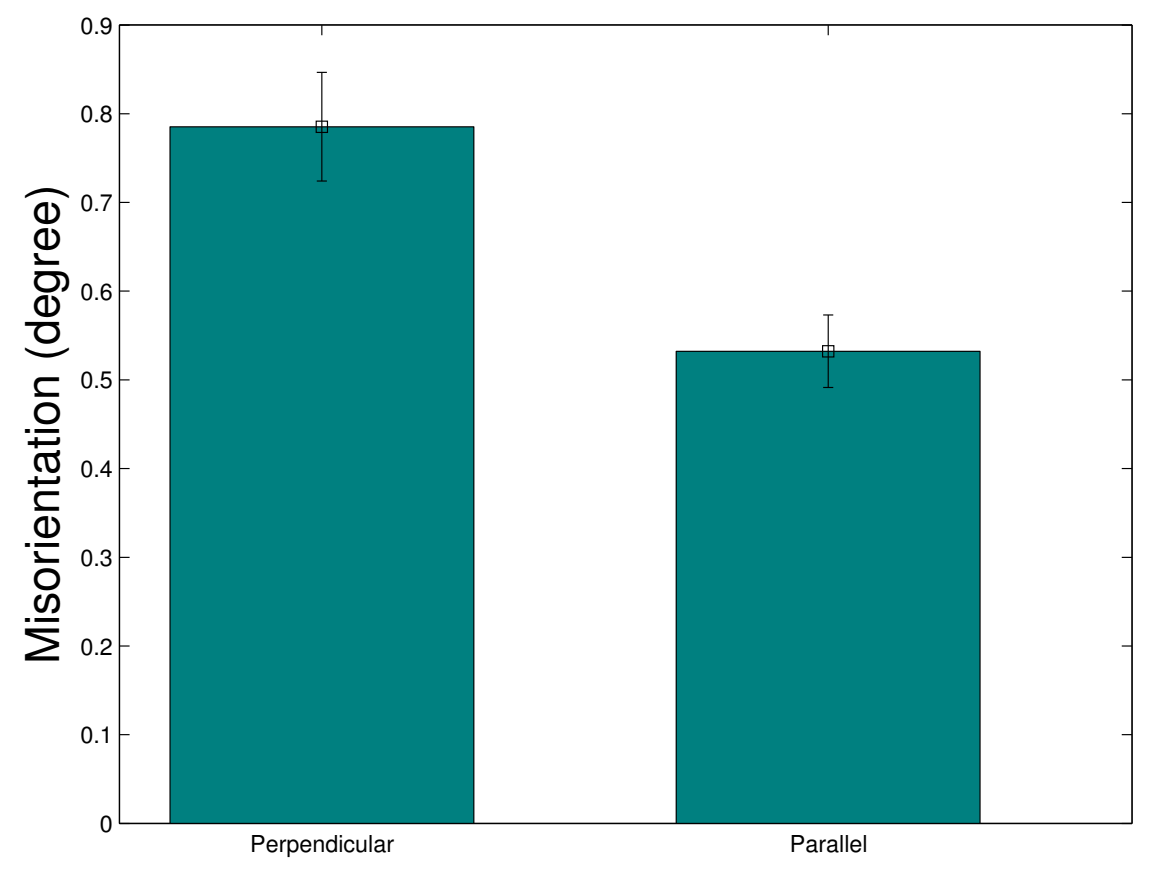

(a)

Perpendicular to GB

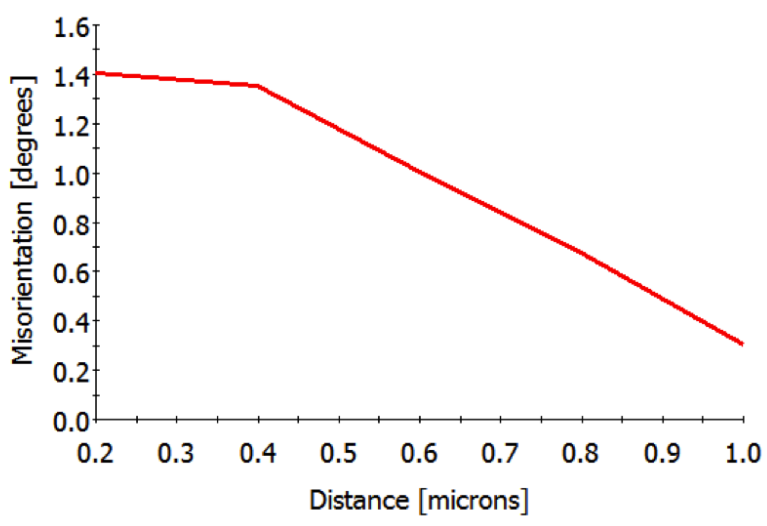

(b)
Parallel to GB

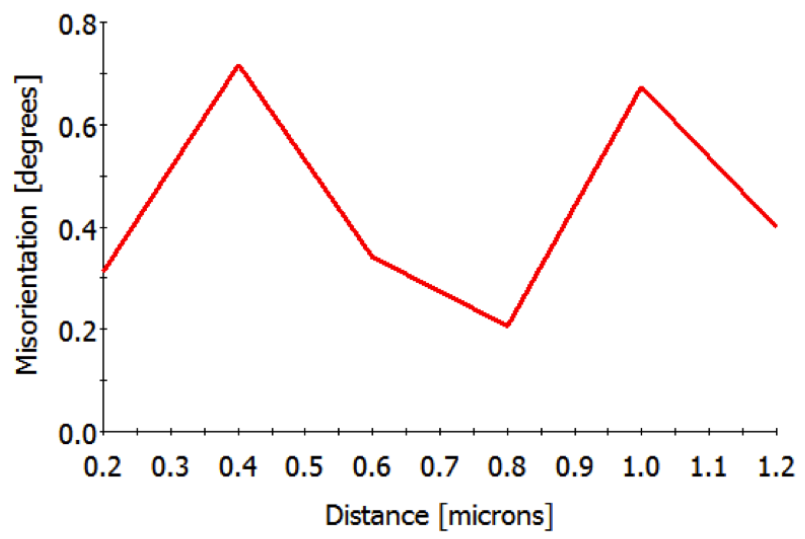

(c)

Figure 9: a) Bar chart showing average misorientation value parallel and perpendicular to grain boundaries b) perpendicular line plot c) parallel line plot.

\section{Conclusion}

Orientation gradients, quantified in terms of KAM, were measured as a function of strain and spatial resolution. Local orientation information from EBSD was analyzed to map KAM vs. Distance from GB for different resolution scans. In both the low and high strain regions, the spatial resolution of the microstructure was varied by changing the step size of the scan. The resolution effect on KAM calculation was thus tested at 
both strain regions. Listed below are the key findings:

1. At low strain, since the scale of the dislocation substructure in the grains is coarse enough, the calculation of KAM is not resolution dependent. Irrespective of the step size used, high values of KAM are seen near the grain boundaries and gradually decrease towards the interior of the grain.

2. However, at high strain, due to the highly misoriented grains and the formation of submicron dislocation structures, it is crucial to choose the correct step size. Using a coarser $(2.5 \mu \mathrm{m})$ step size, high KAM was seen inside the grain whereas using a finer $(0.5 \mu \mathrm{m})$ step size the location of the highest KAM values moved to the grain boundary. Since dislocations generally accumulate at the grain boundary resulting in high misorientation, the latter is believed to be a more accurate observation especially when allied with the computational results.

3. From this study, it can be concluded that one must choose a fine enough step size such that the dislocation substructure is sampled with sufficient resolution. Based on the results reported here, it can be deduced that in pure copper $0.5 \mu \mathrm{m}$ or at least a sub-micron step size should be considered in order to capture all the post deformation features.

\section{Acknowledgments}

This research was supported in part by the US Department of Energy, Office of Basic Energy Sciences, Division of Materials Sciences and Engineering, under award DESC0002001. It was in part supported by Los Alamos National Laboratory's Directed Research and Development (LDRD-DR Project 20140114DR). Support of the Materials Science and Engineering Department at Carnegie Mellon University is acknowledged. Fruitful discussions with Dr. Stuart Wright are also acknowledged.

\section{References}

[1] R. Pokharel, Spatially resolved in-situ study of plastic deformation in polycrystalline copper using high-energy x-rays and full-field simulations (Ph.D. dissertation). Carnegie Mellon University, USA, 2013.

[2] A. J. Schwartz, M. Kumar, B. L. Adams, D. P. Field (Eds.), Electron backscatter diffraction in material science. 2nd ed. New York, NY: Springer Science+Business Media, 2009, pp. 301-315.

[3] S.K Mishra, P. Pant, K. Narasimhan, A.D. Rollett, I. Samajdar, On the widths of orientation gradient zones adjacent to grain boundaries. Scr. Mater., 61 (2009) 273-276.

[4] A. Bhattacharyya Investigating the evolution of grain scale microstructure during large plastic deformation of polycrystalline aluminum (Ph.D. dissertation). Drexel University, USA, 2002.

[5] A.D. Rollett, F. Wagner, N. Allain-Bonasso, D. Field, R.A. Lebensohn, Comparison of gradient in orientation and stress between experiment and simulation. Mate Sci Forum Vols, 702-703 (2012) 463-468. 
[6] Z. Zhao, M. Ramesh, D. Raabe, A.M. Cuitio, R. Radovitzky, Investigation of three-dimensional aspects of grain-scale plastic surface deformation of an aluminum oligocrystal. Int. J. Plast. 24 (2008) 22782297.

[7] D. Raabe, Z. Zhao, S.J. Park, F. Roters, Theory of orientation gradients in plastically strained crystals. Acta Mater., 50 (2002) 421-440.

[8] J. C. Glez, J. H, Driver, Orientation distribution analysis in deformed grains J. Appl. Crystallogr., 34 (2001) 280-288.

[9] M. Kamaya, A. J. Wilkinson, J.M. Titchmarsh, Measurement of plastic strain of polycrystalline material by electron backscatter diffraction Nucl. Eng. Des. 235 (2005) 713725.

[10] Y. Takamaya, J. A. Szpunar, H. Kato, Analysis of intragranular misorientation related to deformation in an Al-Mg-Mn alloy. Mater. Sci. Forum Vols., 495-497 (2005) 1049-1054

[11] A. Ramazani, A. Schwedt, A. Aretz, U. Prahl, Failure initiation in dual-phase steel. Key Eng. Mater., 586 (2014) 67-71.

[12] D.A. Hughes, Microstructure and flow stress of deformed polycrystalline metals. Scripta Metall. Mater., 27 (1992), 969-974.

[13] F. J. Humphreys, M. Hatherly, Recrystallization and related annealing phenomena, First ed.. Pergamon, 1995 , pp. 173-220

[14] R. Pokharel, J. Lind, A. K. Kanjarla, R. A. Lebensohn, S. F. Li, P. Kenesei, R. M. Suter, A. D. Rollett, Polycrystal Plasticity: Comparison between grain scale observations of deformation and simulations. Annu, Rev. Condens. Matter Phys., 5 (2014) 317-46.

[15] S.I. Wright, M.M. Nowell, R. de Kloe, L. Chan, Orientation precision of electron backscatter diffraction measurements near grain boundaries. Microsc. Microanal., 20 (2014) 8521-863.

[16] J. Jiang, T. B. Britton, A. J. Wilkinson, Accumulation of geometrically necessary dislocations near grain boundaries in deformed copper. Philos. Mag. Lett., 92 (2012) 580-588.

[17] J. Jiang, T. B. Britton, A. J. Wilkinson, Measurement of geometrically necessary dislocation density with high resolution electron backscatter diffraction: Effects of detector binning and step size. Ultramicroscopy, $125(2013) 1-9$.

[18] Y. Takayama, J. A. Szpunar, Stored energy and taylor factor relation in an Al-Mg-Mn alloy sheet worked by continuous cyclic bending. Mater. Trans., 45 (2004) 2316-2325.

[19] S. I. Wright, M. M. Nowell, D.P. Field, A review of strain analysis using electron backscatter diffraction. Microsc. Microanal., 17 (2011) 316-2011. 
[20] S.F.F Li, Imaging of orientation and geometry in microstructures: Development and applications of high energy x-ray diffraction microscopy (Ph.D. dissertation). Carnegie Mellon University, USA, 2011.

[21] S. I. Wright, B. L. Adams, Automated lattice orientation determination from electron backscatter Kikuchi diffraction patterns. Text. Microstruc., 14-18 (1991) 273-278.

[22] S. Ghosh, D. Dimiduk (Eds.) Computational methods for microstructure property relationships. Springer Science+Business Media, LLC 2011, pp. 53-97. 